\title{
A study on the effect of process parameters on the joint strength and leak tightness in electromagnetically assisted adhesive Cu-SS tube-to-tube joining through statistical analysis
}

\author{
Deepak Kumar ${ }^{1}$, Sachin D Kore ${ }^{2}$, and Arup Nandy ${ }^{1}$ \\ ${ }^{1}$ Indian Institute of Technology Guwahati \\ ${ }^{2}$ School of Mechanical Sciences, Indian Institute of Technology
}

December 6, 2021

\begin{abstract}
This work studies an improved hybrid joining technique combining electromagnetic forming and adhesive joining to create a leaktight $\mathrm{Cu}-\mathrm{SS}$ tube-to-tube joint named as electromagnetically assisted adhesive joining (EAAJ). An experimental investigation is performed considering three discharge energy $(3.9 \mathrm{~kJ}, 4.4 \mathrm{~kJ}$ and $5.0 \mathrm{~kJ})$, four adhesive application lengths $(20 \mathrm{~mm}$, 15 $\mathrm{mm}, 10 \mathrm{~mm}$ and $5 \mathrm{~mm}$ ), three adhesives (Loctite 638, Loctite 567, and Loctite SI 596) and four curing times (24 hours, 48 hours, 96 hours and 120 hours) as process parameters. The mechanical properties of the joints are investigated using testing techniques like pull-out, compression, and micro-hardness tests. An increase in joint strength is observed with the decrease in adhesive application length and increase in curing time. Maximum joint strength, $90 \%$ of the base copper tube strength, is obtained in the case of Loctite 638, with $5 \mathrm{~mm}$ of adhesive application length, $5.0 \mathrm{~kJ}$ of discharge energy and 96 hours of curing time. Furthermore, a three-way analysis (3-way ANOVA) of variance technique is implemented to calculate the contribution of the three factors (discharge energy, adhesive application length, type of adhesives) on the joint strength. A cohesive and adhesive failure mode combination leading to sliding failure mode is observed as a joint failure mechanism during pull-out and compression testing. A leak testing setup has been developed to investigate the joint's leak tightness by an air pressure decay test. An increment in leak tightness by 1000 times is observed in 638 EAAJ samples compared to samples joined without adhesives. A 3-way ANOVA analysis is also performed to calculate the contribution of different factors on leak tightness of the joint. Micro-hardness is observed to be increased near the joint interface compared to the base metal. Deformation analysis has highlighted the impact of field shaper slit causing a non-uniformity in radial deformation in the circumferential direction and leading to non-uniform circumferential accumulation of adhesive.
\end{abstract}

\section{Hosted file}

Final version to preprint.pdf available at https://authorea.com/users/449535/articles/548042a-study-on-the-effect-of-process-parameters-on-the-joint-strength-and-leak-tightnessin-electromagnetically-assisted-adhesive-cu-ss-tube-to-tube-joining-through-statisticalanalysis 